\title{
La ruta latinoamericana del doctor Faustus: EI lugar sin límites de Donoso y Under the Volcano de Malcolm Lowry
}

The Latin American Route of Doctor Faustus: Donoso's El lugar sin límites and Malcolm Lowry's Under the Volcano

\author{
Felipe Toro Franco \\ Georgetown University \\ ft155@georgetown.edu
}

\begin{abstract}
Estudio sobre El lugar sin límites que propone que la famosa apelación a la obra de Christopher Marlowe por parte de esta obra de José Donoso está mediada por Under the Volcano (1947), de Malcolm Lowry -novela del infierno rural mexicano cuya matriz confesa es el Doctor Faustus de Marlowe. Argumentamos, en suma, que en México de 1965 -donde Donoso escribirá El lugar sin límites, y donde apenas antes de llegar la Revista de la Universidad de la UNAM había publicado un especial conmemorativo dedicado a Bajo el volcán-, José Donoso y Malcolm Lowry se encuentran e intercambian el legado infernal de Christopher Marlowe en tierras latinoamericanas.
\end{abstract}

Palabras clave: José Donoso, Malcolm Lowry, Christopher Marlowe, Infierno latinoamericano.

In this article we argue that José Donoso's famous invocation of Christopher Marlowe's Doctor Faustus in El lugar sin límites (1966) is mediated by the influence of Malcolm Lowry's Under the Volcano (1947) -a novel that addresses the topic of Mexican hell, and which in turn is textually based on Marlowe's drama. We claim that in Mexico, 1965, precisely where Donoso wrote El lugar sin límites, and where, shortly before his arrival, UNAM's Revista de la Universidad had published an issue exclusively devoted to Under the Volcano, José Donoso and Malcom Lowry cross literary paths and negotiate Christopher Marlowe's hellish legacy on Latin American soil.

Keywords: Jose Donoso, Malcolm Lowry, Christopher Marlowe, representations of Latin American Hell. 
A pesar de tratarse de un relato enraizado en el imaginario de la hacienda chilena -con un pueblito moribundo del valle central y una estación de trenes (El Olivo) carcomida por el aislamiento como escenarios fácilmente reconocibles-, El lugar sin límites (1966), de José Donoso, puede paradójicamente leerse como su novela más "mexicana": escrita durante una memorable estadía en casa de Carlos Fuentes, intentando su autor una fuga del espacio simbólico nacional, recrea los cuadros criollistas de principios de siglo desde el pesadillesco trabajo del sueño y la alegoría, echando mano en su epígrafe al mito del Doctor Faustus de Christopher Marlowe: "El infierno no tiene límites, ni queda circunscrito a un solo lugar, porque el infierno es aquí donde estamos y es aquí donde tenemos que permanecer" (Donoso 106). Artes del extrañamiento que de inmediato nos presentan una geografía familiar o, si se quiere, propia -la del valle central con sus antiguos fantasmas de jerarquías de orden rural- desde la dislocación y el desplazamiento, como si la misma cita traducida de Marlowe insinuara una cuña capaz de superar los límites de lo nacional al precio, eso sí, de sumirse en el infierno (traduzcamos: el infierno no queda circunscrito a ningún lugar, ni siquiera a las fronteras de Chile, nos podría decir este Mefistófeles vestido con manta de vicuña, haciéndole verónicas desde México a la búsqueda del color local). No en vano el gesto de leer el campo chileno y sus hileras de viñas interminables mediante Marlowe recuerda a Borges reinterpretando Don Segundo Sombra desde Mark Twain y Kipling ("El escritor argentino y la tradición" 271); y además, Jaime Concha ya hablaba del curioso "destino mexicano" de esta nouvelle de tierra adentro: "El libro aparece en México, fue escrito en la misma casa del autor de MAC [La Muerte de Artemio Cruz], dice en su dedicatoria 'Para Rita y Carlos Fuentes' y, como si fuera su destino, será llevado a la pantalla cinematográfica por un director y con ambientación mexicanos" (96).

¿Es que para El lugar sin límites tampoco es posible salir del infierno, de Comala? Sospecho que si el artilugio satánico en que habitan la Manuela, Pancho Vega y don Alejo se deja trasponer con tal ductilidad en el espacio mexicano en la cinta de Arturo Ripstein (1977) o circular por otras coordenadas culturales -cual figuritas de yeso cambiadas de un lugar a otro- es porque este valle central donosiano y sus esperpentos del agro chilensis, por mucho que en su contenido manifiesto invoquen a grandes señores y rajadiablos, están programados para ser mañosamente traslúcidos; o, dicho de otro modo, han sido dotados de un mecanismo transitivo que les permite moverse simultáneamente por otra geografía y otros textos (el infierno latinoamericano y sus juegos de citas son una extensión transnacional, partes extra partes). Bastará recordar la observación de Rodrigo Cánovas al señalar la similitud a nivel significante de Pancho Vega, macho bruto, hijo de peón que ha ganado su libertad, con Pancho Villa (96); la patente conexión entre el nombre del patrón de fundo donosiano Alejo Cruz y Artemio Cruz, de Carlos Fuentes, como apunta Concha (107); o el espíritu de almas en penas rulfianas que animan los cuerpos de la estación El Olivo (sombra cuya influencia atormentaba al mismo Donoso [Diarios tempranos 268]). Así, con Pancho Vega haciendo las veces de Pancho Villa, la figura de su amigote Octavio, custodio de la sexualidad de su cuñado en la escena que sellará la suerte de la Manuela ("Ya pues compadre, no sea maricón usted también..." [Donoso 207]), especulativamente nos recordará la escritura ensayística de 
otro Octavio (Paz) acerca de las masculinidades mexicanas y sus máscaras impenetrables. Ya veremos cómo terminará la Manuela a manos de estos dos machos tristes, perdida en un laberinto de la soledad, sin que el hilo rojo de su vestido de Ariadna pueda librarla de ser despedazada.

Me atrevería a señalar que entre nosotros la crítica ha escogido principalmente dos caminos para aproximarse a El lugar sin límites y que podríamos resumir a la manera de Proust: por el camino infernal de Marlowe y por el camino mexicano de Carlos Fuentes. Al primero le corresponde el hallazgo de su relación simbiótica con el Doctor Faustus, al que El lugar sin límites está unido desde su título y epígrafe como por un cordón umbilical para producir un modelo a escala del infierno latinoamericano (Cánovas 87; Concha 98). A partir del destino de este arquetipo del individualismo moderno, doctor en teología que, insatisfecho, decide vender su alma al demonio para tener acceso a la magia y a las ciencias ocultas (una biblioteca prohibida, en otras palabras), Donoso esbozaría el espacio rural como un universo regido por deudas y pactos fáusticos: don Alejo con la Japonesa grande y la Manuela; Pancho Vega con don Alejo, al tiempo que todos escuchan las campanadas del reloj que anuncia que no hay plazo que no se cumpla ("The stars move still, time runs, the clock will strike / The devil will come, and Faustus must be damned" [Marlowe 52])1. Por supuesto, la peripecia del Fausto -su transgresión y su destino trágico- está unida indisolublemente a la escena de la escritura y sus recompensas simbólicas: "Lines, circles, letters, and characters- / Ay, these are those that Faustus most desires" (Marlowe 58), reza uno de sus versos introductorios.

Y si bien líneas, círculos y letras son signos propios de la magia, Marjorie Garber indicará que también son principalmente elementos del lenguaje, significantes que nos devuelven la imagen de Fausto condenado por el deseo de escribir o la ambición de convertirse en autor (362). Tal como el Donoso memorialista se ha encargado de situarse respecto de la composición de El lugar sin límites, andando Por el camino mexicano de Carlos Fuentes, enmarañado entre la neurosis y la frustración creativa para dar con el diseño de El obsceno pájaro de la noche (1970). Leyenda que el mismo Donoso ha construido en su Historia personal del boom (1972), y cuya principal escena doméstica, la de nuestro autor, apocado, escribiendo de allegado y a la sombra de la casa grande de Fuentes, cual inquilino, la crítica ha interpretado como

\footnotetext{
1 Para el caso de los paralelismos entre el Doctor Faustus de Marlowe y la Manuela de El lugar sin límites, es Fernando Moreno Turner quien tempranamente los ha elucidado: "Estas dos fuerzas que pugnan en el interior de la figura de Fausto -dios y demonio- también se encuentran en la Manuela. Ella [también] ha establecido un pacto con la Japonesa Grande (indirectamente con don Alejandro), mediante este se convierte en codueña de la casa [...]. Debido a este pacto [...], el personaje permanecerá desde entonces en ese pueblo [...]. En la obra de Marlowe, cuando Fausto debe cumplir su parte del trato, cuando llega la hora de entregar su alma ante la inminencia de la llegada del demonio, el personaje toma conciencia de la individualidad que había perdido [...]. Esto lo conduce a tratar de deshacer el pacto. Pide e implora la bendición de Dios, al mismo tiempo que trata de ocultarse para no ser ubicado por Mefistófeles [...]. Ante el castigo inminente [...], la Manuela, en forma desesperada, trata de salvarse, y perseguida por Pancho y Octavio, huye en busca de don Alejandro [...]. Mientras corre, recuerda la otra parte del pacto [don Alejo le prometió protegerla] [...] y eleva también su plegaria, la que queda solo en su mente" (32-4).
} 
el contenido latente de lo que más tarde surgirá de manifiesto en las relaciones de servidumbre rural que dibuja el texto (la Manuela, Pancho Vega, el hacendado, y don Alejo, patrón de fundo) (Cánovas 95-6):

A las pocas semanas yo ya estaba escribiendo El lugar sin límites. No podía, no debía seguir obsesionado por mi novela larga [El obsceno pájaro de la noche]: con el fin de desembotellarme era necesario escribir otra cosa, quizá más corta $[\ldots]$. Yo tecleaba metido en la sombra del pabellón del fondo del jardín. Al otro lado, en la casa grande [...], Carlos Fuentes escribía Cambio de piel (Donoso 85).

Aquí también la impotencia del Fausto nos ayuda a desgranar el embotellamiento de Donoso: "My blood congeals, and I can write no more" (Marlowe 70).

Ahora bien, todo lector de Proust se preguntaría si finalmente el camino de Marlowe y el camino de Fuentes no terminarán por unirse, con Robert de Saint-Loup desposando a Gilberte Swann o, mutatis mutandis, si alguna vez el Fausto habitará el infierno mexicano de Rulfo. Dicho de otro modo, se trata de inquirir por qué la autoría en esta, su "novela mexicana" por excelencia -su "destino mexicano", diría Concha- ha escogido precisamente a Christopher Marlowe como su Virgilio retórico. Quizá aquí valga la pena recordar que el título con el que Donoso trabajó hasta último minuto -incluso hasta después de entregada la nouvelle a la editorial Joaquín Mortiz- fue Ríe el eterno lacayo, que sospecho proveniente de los versos de T.S. Eliot de The Love Song of J. Alfred Prufrock (no por nada precedido de un epígrafe del infierno del Dante): "And I have seen the eternal Footman hold my coat, and snicker, / And in short, I was afraid" (6). Y que antes, en sus diarios de escritor anotaba ya instalado en México: "[f]acilísimo buscar un título para esto [El lugar sin límites] en una cita shakespereana de una de las comedias de transvestismo" (Donoso, Diarios tempranos 263).

Por cierto, estamos nuevamente tentados de ver, entre los renglones torcidos de la escena de salón de Prufrock, la fantasía del eterno lacayo Donoso -que demoníacamente espera reír al último y reír mejor- mientras sostiene el abrigo de un Fuentes aterrado; y a leer el desplazamiento que va de la comedia de Shakespeare a la tragedia de Marlowe desde la identificación de Donoso con el precursor eclipsado por el genio (Marlowe, que no Shakespeare, agazapado en el segundo plano de una muerte prematura). Vale, ¿pero qué papel juega aquí el Doctor Faustus, o más bien, de dónde surge el famoso epígrafe de la novela, que vendrá a constituirse -de manera tardía, eso sí- en la viga maestra de su hechura infernal? De eso se trata precisamente nuestra tesis: la apropiación del Doctor Faustus por parte de Donoso -proponemos- no viene simplemente de la biblioteca de sus años de estudiante de literatura inglesa en Princeton, sino que su invocación a la obra de Marlowe está mediada por la lectura de Under the Volcano (1947), de Malcolm Lowry: otra alegoría del infierno mexicano escrita por un extranjero, bajo el signo del Doctor Faustus.

Esta vez, el infierno es Cuernavaca (Quauhnahuac), y Fausto está representado por el alcoholizado excónsul británico Geoffrey Firmin, el día 
de muertos de 1938 (su último día de vida). La obra parte con una escena de lectura, con la imagen de Jacques Laurelle un año después de los acontecimientos, leyendo una antología de teatro isabelino que Firmin le había prestado: "M. Laurelle had opened the book of Elizabethan plays [...] and for a moment he sat oblivious of his surroundings, gazing at the words that seemed to have the power of carrying his own mind downward into a gulf, as in fulfilment on his own spirit of the threat Marlowe's Faustus had cast at his despair" (Lowry 35). Movidas bajo el patrón de la desesperación fáustica, en palabras de Ronald Walker y Leigh Holt, las simetrías entre Marlowe y Lowry parten desde las primeras palabras del cónsul -tomadas directamente del Doctor Faustus- hasta que es muerto a tiros y su cuerpo es arrojado por los "diablos" (la policía clandestina) al Tártaro (una barranca a los pies del volcán Popocatépl), pasando por los tragos tomados en ni más ni menos que el Hotel Fausto (Walker y Holt 112). Una única jornada de doce horas, desde las siete de la mañana hasta las siete de la noche, círculo perfecto del delirium tremens de Geoffrey Firmin, que se cierra al ser confusamente ajusticiado en una cantina rural y terminar compartiendo una fosa común con un perro muerto. Del mismo modo que el arco narrativo de El lugar sin límites, que se abre y se cierra con un solo día de la Manuela (suponemos también que el último), desde que se despierta cinco para las diez de la mañana hasta que la Japonesita -su hija- se va a acostar cerca de las cinco de la madrugada sin saber que sobre su padre se han abalanzado, cual jauría, Pancho Vega y Octavio. "I hold to the number twelve", dirá Lowry en el prefacio a Under the Volcano, "It is as if I heard a clock sounding midnight for Faust, and when I think of the slow progression of the chapters, I feel that neither more nor less than twelve should satisfy me" ("Preface" 8). ¿Adivinará el lector con cuántos capítulos cuenta nuestro marloviano El lugar sin límites? Doce, marcados también a punta de reloj -"[f]altaba media hora para la misa" (115) o "[c]inco minutos hasta la casa de la Ludovina, un cuarto de hora para buscar el hilo, y cinco minutos para cualquier cosa [...]" (115-6)-, hasta llegar in crescendo al momento en que la Manuela ("virgen y mártir" [123]) es ultimada a golpes por Pancho Vega, Pancho Villa.

No será la única coordenada temporal que en El lugar sin límites nos haga eco de la arquitectura fáustica de Under the Volcano. En la novela de Lowry, el día de muertos de 1939 empieza con el regreso de Yvonne a Cuernavaca, quien después de un año de ausencia vuelve para intentar salvar su matrimonio con el cónsul y quizá sacarlo de su borrachera (un año sin Yvonne en el que Firmin le ha escrito cartas de amor nunca enviadas y que finalmente terminarán guardadas en el volumen de teatro isabelino donde está el Doctor Faustus de Marlowe). Y si Under the Volcano comienza con el regreso de Yvonne, la novela de Donoso lo hará con Pancho Vega volviendo a El Olivo, justamente después de un año de ausencia, como si el engranaje del reloj del Fausto del valle central chileno estuviera en sincronía con el tempo de Cuernavaca: "[e]n cuanto le dijeron que Pancho Vega andaba en el pueblo, le entró la tentación de sacar su vestido otra vez. Hacía un año que no lo tocaba" (109); "Perdido anda [Pancho] desde hace un año" (120). Además, curiosamente, cuando ya se encuentre instalado en Cuernavaca en 1965, Donoso coqueteará con la idea de escribir un cuento de especular parecido al destino de Yvonne y su pasado como actriz de Hollywood ("So Yvonne Constable, at twenty-four, is well on the way for the second time to becoming 
a star. -But Yvonne Constable had not become a star for the second time" [Lowry 274]), cuyo título irónicamente habría sido el de "El paraíso": "Una actriz americana, güera y que ha sido símbolo de lo sexual, tiene un calamitoso fracaso profesional en Hollywood y se retira de la carrera con una decente cantidad de dinero y se viene a encerrar en una casa en Cuernavaca, donde hace amistades y lleva una vida divertida pero retirada: mundo de seres fracasados en el paraíso de Cuernavaca [...]" (Diarios tempranos 387).

Eso sí, mientras en Under the Volcano la pareja está compuesta entre Yvonne y Geoffrey -que giran desesperados como Paolo y Francesca en los primeros círculos del infierno del Dante-, en El lugar sin límites los amantes son la pareja monstruosa, sin calce posible, entre la Manuela y Pancho Vega, que se reencuentran en un abrazo de erotismo y violencia (la destrucción del cuerpo del deseo a mitad de la noche) cuando la Manuela intenta cruzar las viñas para llegar a la casa de don Alejo: "[l]os cuerpos calientes retorciéndose sobre la Manuela que ya no podía ni gritar, los cuerpos pesados, rígidos, los tres una sola masa viscosa retorciéndose como un animal fantástico de tres cabezas y múltiples extremidades heridas e hirientes [...], el cuerpo endeble de la Manuela que ya no resiste, quiebra bajo el peso [...]" (209-10). Apoteosis de violencia y exceso, tanto Donoso como Lowry al llegar la hora señalada -la hora en que Fausto es llevado al infierno, sus últimas páginas- nos ofrecen muertes brutales, con rostro de bestialidad (por lo pronto, las muecas perrunas del can Cerbero en Donoso y el perro en la barranca del Popocatépl en Lowry). Yvonne morirá intentando cruzar un bosque (como la Manuela intentando cruzar las viñas) para llegar a la cantina donde se encuentra Geoffrey, aplastada por un caballo desbocado (¿otro animal fantástico que "la quiebra bajo su peso"?), mientras escuchamos su acezante monólogo interior, un éxtasis negro in articulo mortis ("[t]hey were in a dark wood, she heard the wind and the rain rushing through the forest and saw the tremours of lightning shuddering through the heavens and the horse -great God, the horse- and would this scene repeat itself endlessly and forever? -the horse, rearing, poised over her, petrified in midair [...]" [349]).

Y Geoffrey será ejecutado a manos de la policía clandestina mexicana, cual víctima sacrificial invertida: un Cristo borracho, tal como la Manuela -el Emmanuel, dios con nosotros (Millares 63)- será "virgen y mártir" (uno de los policías le preguntará a Geoffrey: "You are Juden?" [Lowry 373]). Agreguemos que si El lugar sin límites gira en torno al prostíbulo rural de la Manuela, el cónsul Firmin encontrará la muerte a causa de un malentendido en una esperpéntica cantina y burdel Ilamado El Farolito (el burdel es el espacio del clímax): "'Quiere María?' a voice spoke softly behind him. At first he saw the shapely legs of the girl who was leading him, now by the constricted power of aching flesh alone, of pathetic trembling yet brutal lust, through the little glass-paned rooms, that grew smaller and smaller, darker and darker [...]" (Lowry 362). Este es un Farolito que paradójicamente no alumbra (es una pura oscuridad existencial); Farolito que, además, está situado en Parián y en las cercanías de Tomalín, pueblo rural ficticio que Lowry emplaza en las cercanías de Cuernavaca; un pueblo cuyo destino -sueños frustrados de modernidad y energía hidroeléctrica- nos recuerda, por asociación libre, a la descripción de El Olivo sin luz: "Once, perhaps, in hacienda days, Tomalín had held some irrigational importance. Then, after the burning of sugar 
plantations, schemes, cleavable and lustrous, evolved for a spa, were abandoned sulphurously. Later, vague dreams of hydro-electric power hovered in the air, though nothing had been done about them" (Lowry 296). Sulfúrico o infernal abandono en que la hacienda mexicana y la hacienda chilena se miran en el espejo: "Tanto que habló de la electrificación con don Alejo. Y nada. Después anduvieron diciendo que el camino pavimentado, el longitudinal, iba a pasar por El Olivo mismo, de modo que se transformaría en un pueblo de importancia [...]. Pero después le dijeron la verdad, don Alejo, creo, que el trazado del camino pasaba a dos kilómetros del pueblo [...]" (138).

Especulativamente podríamos establecer un juego de correspondencias entre estos dos mapas. Del lado de Lowry, está Cuernavaca y, en su periferia rural imaginaria, a los pies del volcán Popocatepl, Tomalín y Parián, cobijándose en este último pueblo el burdel de El Farolito -entre Tomalín y Parián hay un bosque, que es donde muere Yvonne embestida por el caballo-. Del lado de Donoso está Talca, y en su periferia, el abandonado e imaginario pueblito de El Olivo, donde está el prostíbulo de la Manuela -entre el pueblo y la casa de don Alejo están las viñas y las zarzamoras, que es donde la Manuela es atacada por Pancho Vega y Octavio-. Proporciones: ¿será que Cuernavaca es a Talca como Parián y Tomalín son a El Olivo, como el Farolito es al prostíbulo de la Manuela, y como el bosque al pie del volcán es a las viñas y a las zarzamoras al pie de la cordillera? Todos bajo el signo de la tragedia de la modernidad del terruño latinoamericano -sulfúrico abandono-, invocando la protección narrativa de Christopher Marlowe, cada uno con doce capítulos que describen el arco de la última jornada mortal de un esperpento. En El lugar sin límites, Donoso, Lowry y Marlowe se dan cita en el infierno. O, si se quiere, para que Marlowe llegara al valle central chileno, las rutas de Donoso y Lowry tuvieron que encontrarse en México.

¿Pero dónde se cruzan Donoso y Lowry? Si por una parte sabemos que Lowry comenzó a escribir Under the Volcano en Cuernavaca en 1936, Donoso llegará a México a fines de 1964 (casi treinta años después) invitado a asistir a un congreso literario por Carlos Fuentes, en cuyo jardín habría desgajado un episodio autónomo de El obsceno pájaro de la noche que "[e]n dos meses quedó convertido en El lugar sin límites" (Donoso, Historia personal del boom 85). Y si bien la traducción al español de Under the Volcano fue tardía (Bajo el Volcán, traducida por Raúl Ortiz y Ortiz, verá la luz en 1964), la pintura de época de Sharae Deckard nos hace suponer que Lowry ya ocupaba un lugar principal en la biblioteca mexicana que recibió a Donoso:

Although Juan Ortiz's [sic] Spanish translation, Bajo el volcán, did not appear until several decades after the U.S. publication, the novel was widely read and praised by Latin American intellectuals in the mid-century. The Mexican literary movement of the 1950s and 1960s known as La Generación de Medio Siglo dedicated an issue of their seminal journal, Revista de la Universidad, to Malcolm Lowry; Carlos Fuentes established a relation between Lowry's novel and a mythic Mexican identity: "I consider it one of the best Mexican novels... Lowry is a wonderful writer. Maybe, even, a Mexican writer"; and Octavio Paz 
positioned Lowry in a line of 'Mexican' writers leading from D.H. Lawrence to Juan Rulfo (Deckard 68).

De hecho, Donoso arriba a México el mismo año de la traducción de Lowry al español y apenas un mes después de que la Revista de la Universidad-de la UNAM- dedicara en su totalidad el número de noviembre de 1964 a Under the Volcano, que en no pocos casos interpretaba el texto de manera documental, acompañando el texto con fotografías de los lugares de Cuernavaca que habían inspirado la obra: "Por excepción a nuestra norma, hemos dedicado un número entero a la obra de un solo escritor [...]. En primer término, hemos pensado que Malcolm Lowry no es un escritor cualquiera [...]. México era para Lowry una verdadera obsesión. Lo mexicano se presenta en sus escritos con una amorosa violencia raras veces igualada en un autor extranjero" (García Terrés 3).

\section{UNIVERSIDAD DE MEXICO \\ NOVIEMBRE 1964 \\ LA OBSESIÓN DE MÉXICO \\ MALCOLM LOWRY \\ PAPELES INÉDITOS Y NUEVAS APROXIMACIONES}

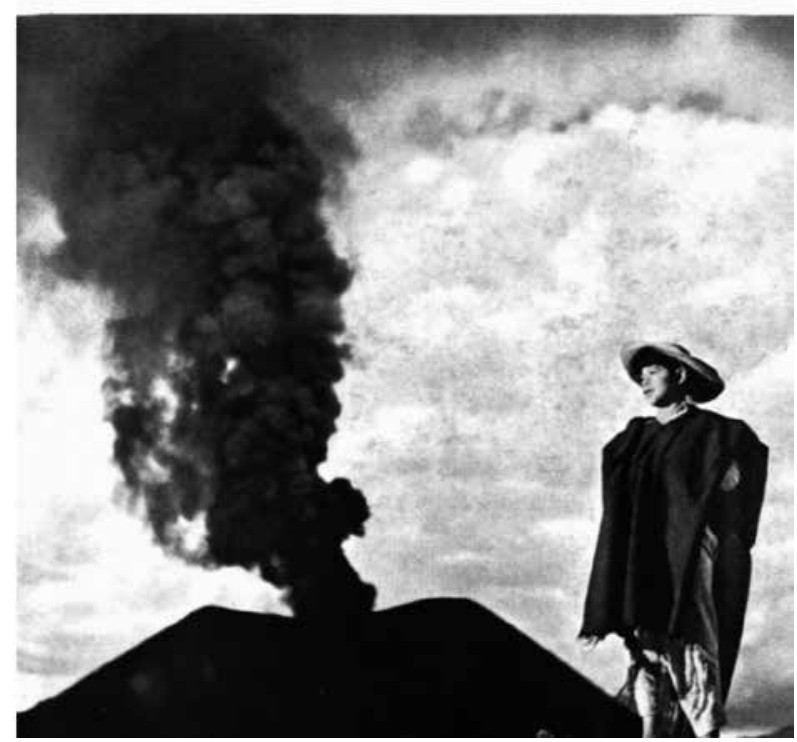

Portada del número de la Revista de la Universidad dedicado a Lowry (noviembre de 1964)2.

2 Agradezco la gentileza de la Coordinación editorial de la Revista de la Universidad por su autorización para reproducir la imagen de la portada. 
¿Habrá encontrado Donoso en la biblioteca de Fuentes (la biblioteca oculta o prohibida del Fausto) un Bajo el volcán recientemente traducido por Ortiz o el último ejemplar de la Revista de la Universidad (de la que Fuentes por lo demás era colaborador)? Por supuesto que no lo sabemos. Lo que sí sabemos es que Fuentes fue un fervoroso promotor de Malcolm Lowry, al punto de que en el verano londinense de 1967 intentaría persuadir a Guillermo Cabrera Infante (además de que leyera con urgencia la novela) de escribir juntos el guión cinematográfico de Under the Volcano que habría estado interesado en filmar Luis Buñuel (Souza 103); y en La nueva novela hispanoamericana, Lowry -junto a Faulkner, Golding y Broch- representaba su fórmula de mito, lenguaje y estructura que prefiguraba como un faro la novela del porvenir: "En Lowry no son las relaciones de clase de Yvonne y el Cónsul lo importante, sino el mito del paraíso perdido y su representación trágica y fugaz del amor" (Fuentes 19).

En este sentido, vale la pena revisitar, desde la presencia fantasmal de Lowry en la biblioteca de Fuentes, la escena del jardín de Historia personal del boom citada más arriba, aquella en que Donoso "tecleaba [El lugar sin límites] metido en la sombra" mientras Fuentes, "con Las estaciones de Vivaldi puesto a todo lo que daba el tocadiscos [..., ] escribía Cambio de piel" (85). Porque -como han señalado José Miguel Oviedo y Suzanne Levine ${ }^{3}$ uno de los modelos de Cambio de piel, "reconocibl[e] y explícit[o]" (Oviedo 6 ), es precisamente Under the Volcano de Lowry. Ya sabemos al menos por qué ríe el eterno lacayo: en un retorcido juego de rivalidad y envidia, Donoso desvalija la biblioteca de la que se alimenta en ese momento la obra de Fuentes (el pathos mexicano de Lowry); desde las sombras del patio de atrás cual Luzbel rebelde se apodera de sus tesoros (burlando las leyes de la hospitalidad), y reutiliza sus materiales para dar vida a su propio artificio (una nouvelle que en tono menor se ofrece como interferencia del comercio de citas de Fuentes y como reino mínimo ganado para las almas condenadas). A la imagen prometeica del Pájaro devorándole las tripas al creador, respondemos con su revés, con Donoso robando el fuego sagrado mexicano.

Finalicemos con una última adenda conjetural, provista por sus diarios de escritor recién publicados, donde Donoso en 1965 -emplazado esta vez en Cuernavaca tal como Lowry treinta años atrás- todavía sigue llamando a su novela Ríe el eterno lacayo, incluso ya entregado el manuscrito a la editorial Joaquín Mortiz (Diarios tempranos 609). No es esta su última versión -de lo contrario estaríamos leyéndolo a la luz de J. Alfred Prufrock y T.S. Eliot y no del infierno de Marlowe-, y de ahí que sospechemos que es en la Cuernavaca de Lowry donde El lugar sin límites adquirió su forma definitiva. Así lo señalaba un testimonio de Vicente Leñero (que hasta ahora uno hubiera estado

\footnotetext{
3 Para un análisis comparativo entre la novela de Fuentes y la de Lowry, remito a "The Pyramid and the Volcano: Carlos Fuentes' Cambio de piel and Malcolm Lowry's Under the Volcano", donde Suzanne Levine señala: "The principal action in both novels significantly takes place on a day that celebrates the myth of cycle of Life and Death. Under the Volcano begins on the Day of the Dead in November 1939, one year after which the principal action occurs. In Cambio de piel the story [...] takes place on [...] Palm Sunday. At these special times characters in each work descend into an abyss of darkness and death" (26).
} 
tentado a descartar por impreciso y por no coincidir con el de Donoso en Historia personal del boom):

De la colonia Cuauhtémoc, los Donoso se trasladaron a una hermosa casa alquilada en Cuernavaca, en el rumbo de Acapatzingo [...]. Donoso ya tenía el título de su novela, El obsceno pájaro de la noche, pero la novela continuaba frenada. Para salir del empalamiento decidió desprender de su polifonía en proceso una de las muchas historias que se había imaginado, y la escribió de golpe [...]: El lugar sin límites (Leñero 50).

Imaginamos entonces a Lowry en 1936 invocando al Fausto de Marlowe en Cuernavaca para dar vida a la jornada trágica de Geoffrey Firmin, circular como el infierno; y a Donoso en 1965 invocando al Fausto de Marlowe en Cuernavaca para terminar de diagramar el día fatal de la Manuela, circular como la arquitectura de Lowry.

\section{Obras citadas}

Borges, Jorge Luis. "El escritor argentino y la tradición". Obras completas: 1923-1972. Ed. Carlos V. Frías. Buenos Aires: Emecé Editores, 1974. 267-274.

Cánovas, Rodrigo. "Una relectura de El lugar sin límites, de José Donoso". Anales de Literatura Chilena 1 (2000): 87-99.

Concha, Jaime. "Cruces hispanoamericanos: Fuentes, Donoso y El lugar sin límites". Revista de Crítica Literaria Latinoamericana 53 (2001): 95-113.

Deckard, Sharae. Paradise Discourse, Imperialism, and Globalization: Exploiting Eden. London: Routledge, 2010.

Donoso, José. Diarios tempranos. Donoso in progress, 1950-1965. Ed. Cecilia García Huidobro. Santiago: Ediciones Universidad Diego Portales, 2016. El lugar sin límites. Ed. Selena Millares. Madrid: Ediciones Cátedra, 2010. Historia personal del boom. Santiago: Andrés Bello, 1987.

Eliot, T.S. "The Love Song of J. Alfred Prufrock". Collected Poems 1909-1962. New York: Harcourt, Brace \& World, Inc., 1963. 3-7.

Fuentes, Carlos. La nueva novela hispanoamericana. México, D.F: Editorial Joaquín Mortiz, 1972.

Garber, Marjorie. "Writing and Unwriting in Doctor Faustus". Doctor Faustus.

Christopher Marlowe. Ed. David Scott Kastan. New York: Norton, 2005. 361-372.

García Terrés, Jaime. "La feria de los días". Revista de la Universidad de México 3. Vol. XIX (1964): 3.

Leñero, Vicente. "Injurias y aplausos para José Donoso". La gente así. México D.F: Alfaguara, 2008. 48-56.

Levine, Suzanne. "The Pyramid and Volcano: Carlos Fuentes' Cambio de piel and Malcolm Lowry's Under the Volcano". Mester 11.1 (1982): 25-40.

Lowry, Malcolm. "Preface to a Novel". Malcolm Lowry: The Man and His Work. Ed. George Woodcock. London: Black Rose Books, 2007. 3-8. Under the Volcano. New York: Harper Collins, 2007.

Marlowe, Christopher. Doctor Faustus. Ed. David Scott Kastan. New York: Norton, 2005. 
Millares, Selena. "Introducción". El lugar sin límites. José Donoso. Ed. Selena Millares. Madrid: Ediciones Cátedra, 2010. 9-91.

Moreno Turner, Fernando. "La inversión como norma: a propósito de El lugar sin límites". Cuadernos Hispanoamericanos 295 (1975): 19-42.

Oviedo, José Miguel et al. "Carlos Fuentes at UCLA: An Interview". Mester 11.1 (1982): 3-15.

Souza, Raymond D. Guillermo Cabrera Infante: Two Islands, Many Worlds. Austin: U of Texas P, 1996.

Walker, Ronald y Leigh Holt. "The Pattern of Faustian Despair: Marlowe's Hero and Under the Volcano". Apparently Incongruous Parts: The Worlds of Malcolm Lowry. Ed. Paul Tiessen. Metuchen, NJ: Scarecrow Press, 1990. 110-28. 
